\title{
Perfil do Paciente Ostomizado e Complicações Relacionadas ao Estoma*
}

\author{
Profile of the Pacient with Stoma and Complications Related to Stoma
}

\author{
CARLOS HENRIQUE MARQUES DOS SANTOS ${ }^{1}$, MARCELO MATOS BEZERRA ${ }^{2}$, FERNANDO MÁRCIOMATOS \\ BEZERRA $^{3}$, BIANCARAHALPARAGUASSÚ ${ }^{4}$
}

${ }^{1}$ Titular da Sociedade Brasileira de Coloproctologia; ${ }^{2}$ Médico residente em cirurgia geral do HRMS; ${ }^{3}$ Acadêmicos de Medicina da Universidade Federal de Mato Grosso do Sul, UFMS, Campo Grande, MS - Brasil.

\begin{abstract}
SANTOS CHM; BEZERRA MM; BEZERRA FMM; PARAGUASSÚ BR. Perfil do Paciente Ostomizado e Complicações Relacionadas ao Estoma. Rev bras Coloproct, 2007;27(1): 016-019.

RESUMO: Objetivo: elaborar o perfil dos pacientes ostomizados e as complicações relacionadas ao estoma. Material e Método: estudo retrospectivo em que foram analisados prontuários dos pacientes cadastrados no Programa de Ostomizados do Centro de Especialidades Médicas da Prefeitura Municipal de Campo Grande-MS. Resultados: foram avaliados 178 prontuários (56.7\% homens e $43.3 \%$ mulheres). A média de idade entre os pacientes foi de 46.8 anos para o sexo masculino e de 54.6 anos para os do sexo feminino. Dentre as ostomias, foram encontradas 152 colostomias $(85.4 \%)$, 21 ileostomias (11.8\%) e 5 urostomias $(2.8 \%)$. 0 principal motivo para confecção dos estomas foi a neoplasia maligna (46.6\%), seguido do trauma abdominal (7.3\%), e do desvio de trânsito intestinal devido a úlceras de pressão (6.7\%). Adaptação inadequada da placa ao estoma foi encontrada em 90 pacientes (50.6\%). Complicações do estoma foram encontradas em 103 pacientes $(57.9 \%)$, dentre elas, dermatite peri-estomal (28.7\%), estoma plano (18.6\%), hérnia para-colostômica $(\mathbf{1 0 . 7 \%})$ e retração do estoma (10.1\%). Conclusões: No grupo estudado, o principal motivo para a confecção de ostomia foi a neoplasia de retossigmóide e canal anal. A complicação mais comum foi a dermatite periestomal.
\end{abstract}

Descritores: ostomia, morbidade, complicações pós-operatórias, estatística \& dados numéricos, perfil de saúde.

\section{INTRODUÇÃO}

A confecção de um estoma é um procedimento comum, realizado por diversas especialidades cirúrgicas, sobretudo em situações de urgência, visando à redução da morbimortalidade pós-operatória ${ }^{1}$. Apesar de comumente realizado, tal procedimento é potencialmente acompanhado de complicações que na maioria das vezes são subestimadas ${ }^{2}$. Estudos mostram taxas de complicações relacionadas aos estomas que variam de 21 a $60 \% \%^{2,3,4}$.

Grande parte de tais complicações podem ser evitadas com o planejamento do local de confecção do estoma e com o uso de técnica cirúrgica adequada. Principalmente nos casos de estomas definitivos, uma maior atenção na sua confecção, que ocorre normal- mente ao final do procedimento cirúrgico, poderá proporcionar melhor qualidade de vida ao paciente, com menores taxas de complicações ${ }^{5}$.

As principais complicações relacionadas aos estomas incluem a adaptação inadequada da placa de ostomia, devido à má localização do estoma na parede abdominal, dermatite periostomal, necrose isquêmica, retração, prolapso, estenose, fístula periostomal, hérnia periostomal, abscesso periostomal e câncer. Como manifestações sistêmicas, podem ocorrer distúrbios hidroeletrolíticos, em estomas de alto débito, e anemia, em casos de sangramento de varizes localizadas no estoma ${ }^{6}$. Além das complicações citadas, existe ainda, nos casos de ostomias temporárias, a morbimortalidade relacionada ao procedimento de fechamento dos mesmos.

\footnotetext{
* Estudo realizado no Serviço de Cirurgia Geral do Hospital Regional de Mato Grosso do Sul, HRMS, Campo Grande, MS - Brasil. 
Perfil do Paciente Ostomizado e Complicações

Relacionadas ao Estoma

Carlos Henrique Marques dos Santos e Cols.
Vol. 27

\section{OBJETIVO}

O presente estudo tem como objetivo avaliar as complicações relacionadas aos estomas e o perfil dos pacientes ostomizados, cadastrados no Programa de Ostomizados do Centro de Especialidades Médicas da Prefeitura Municipal de Campo Grande-MS, que atende além da capital, Campo Grande, outros 12 municípios do Estado de Mato Grosso do Sul.

\section{MATERIAL E MÉTODO}

Estudo retrospectivo, em que, através da análise dos prontuários dos pacientes ostomizados, cadastrados no Programa de Ostomizados do Centro de Especialidades Médicas da Prefeitura Municipal de Campo Grande-MS, foram colhidas informações acerca da idade e sexo dos pacientes, além de tipo, motivo e eventuais complicações dos estomas. Foram excluídos da análise prontuários incompletos e pacientes que, apesar de cadastrados, não participavam mais do programa, devido a óbito, mudança de domicílio ou outras causas. Um total de 178 prontuários foram avaliados.

\section{RESULTADOS}

Dos 178 pacientes incluídos na análise, 101 eram do sexo masculino (56.7\%) e 77 eram do sexo feminino $(43.3 \%)$, conforme demonstrado na figura1. A média de idade entre os pacientes do sexo masculino foi de 46.8 anos e entre os do sexo feminino foi de 54.6 anos (Figura 2).
Dentre as ostomias, foram encontradas 152 colostomias (85.4\%), 21 ileostomias (11.8\%) e cinco urostomias (2.8\%). Com relação ao motivo do estoma, a principal causa foi neoplasia maligna, com um total de 102 casos (67.1\%), sendo que o câncer de retossigmóide e canal anal perfizeram $63.7 \%$ desses casos. Como segunda causa mais prevalente, foi encontrado trauma abdominal, com 13 casos (7.3\%), e terceira, o desvio de trânsito intestinal devido a úlceras de decúbitos por pressão, com 12 casos (6.7\%), conforme demonstrado na figura 3.

Das 152 colostomias, 48 (31.6\%) eram em alça e $104(68.4 \%)$ eram terminais. Dentre as 21 ileostomias, cinco eram em alça $(23.8 \%)$ e 16 eram terminais (76.2\%).

Adaptação inadequada da placa ao estoma foi encontrada em 90 pacientes (50.6\%). Complicações do estoma foram encontradas em 103 pacientes $(57.9 \%)$, dentre as quais, as mais prevalentes foram: dermatite $(28.7 \%)$, estoma plano $(18.6 \%)$, hérnia periostomal $(10.7 \%)$ e retração do estoma $(10.1 \%)$.

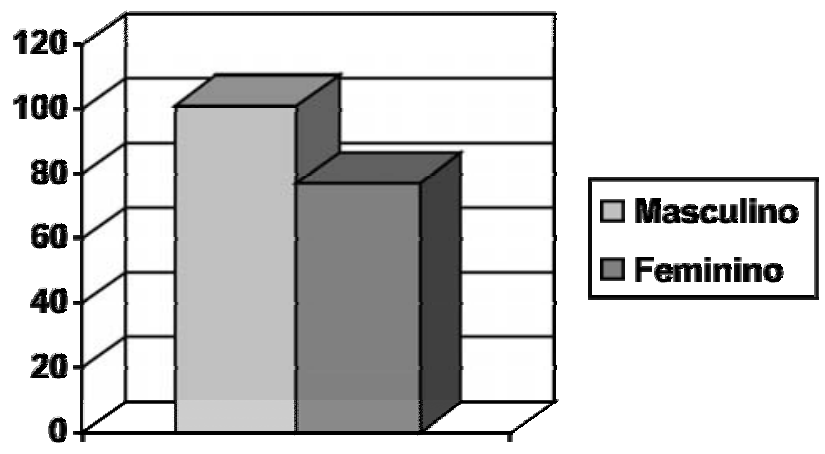

Figura 1 - Distribuição dos pacientes quanto ao sexo.

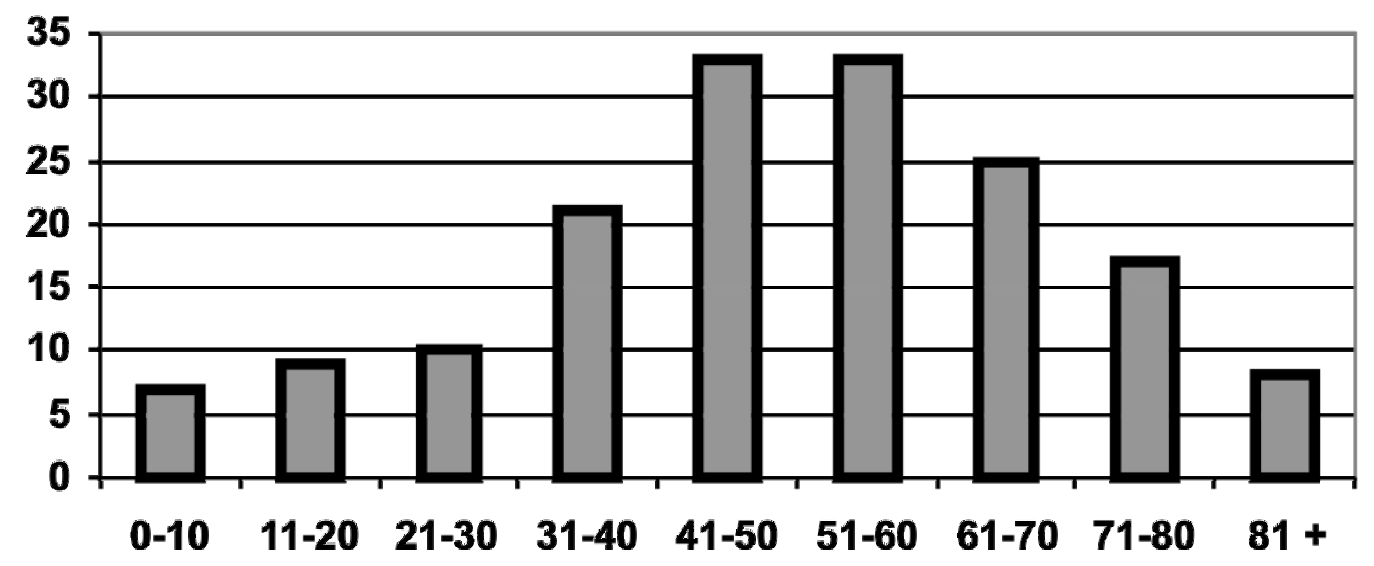

Idade (anos)

Figura 2 - Distribuição dos pacientes quanto à idade. 

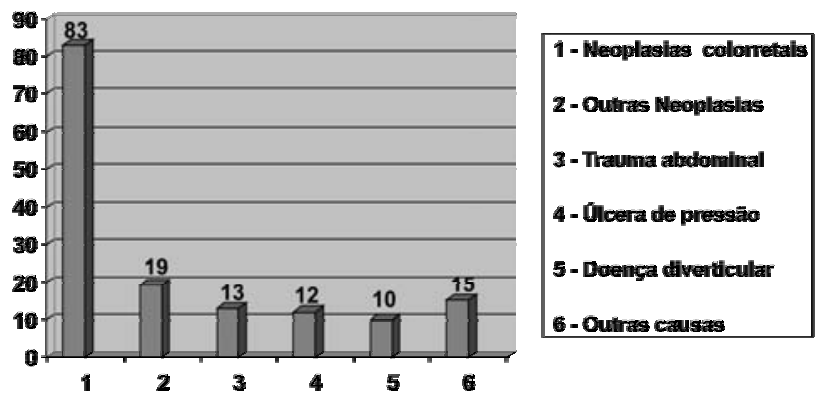

Figura 3 - Indicação dos estomas.

As principais complicações tardias relacionadas aos tipos de estomas estão demonstradas na tabela 1 .

\section{DISCUSSÃO}

O principal motivo para realização do estoma foi a neoplasia maligna, principalmente de retossigmóide (46.6\%), seguido do trauma abdominal (7.3\%), e do desvio de trânsito intestinal devido a úlceras de pressão $(6.7 \%)$. Esta distribuição foi também observada recentemente por Duchesne et al. ${ }^{2}$. Já outros autores ${ }^{1,7,8}$, em estudos mais antigos, descrevem o trauma como a principal indicação de ostomia. Tal diferença ocorre, provavelmente, devido à tendência atual da anastomose primária para tratamento das lesões traumáticas do cólon. Acompanhando a maior prevalência do câncer colorretal como indicação de estomas, os pacientes do presente estudo apresentam idade média acima do observado em outras publicações ${ }^{1,8}$.

A má adaptação da placa do estoma à pele ocorreu em metade dos pacientes. Isso se deve principalmente à escolha inadequada do local de confecção do estoma na parede abdominal, situando-se próximo a depressões, pregas cutâneas, proeminências ósseas, entre outros acidentes anatômicos, permitindo o vazamento do conteúdo drenado pelo estoma. Estudos demonstram que a localização inadequada do estoma é mais comum em cirurgias de urgência, denotando a importância do planejamento pré-operatório ${ }^{9}$. Esta complicação varia de $13 \%$ nas cirurgias eletivas a $31 \%$ nas cirurgias de urgência ${ }^{9}$. Outros autores não encontraram tal diferença ${ }^{2,5}$.

De maneira global, foi demonstrado no presente estudo que $57.9 \%$ dos pacientes apresentaram alguma complicação local relacionada ao estoma. Tal resultado foi também demonstrado por outros autores, que encontraram complicações locais variando de 16 a $90 \%{ }^{2,9,10}$

Dentre as complicações, a mais comumente encontrada foi a dermatite periostomal (28.7\%), assim como demonstrado por outros autores ${ }^{5,9,10}$. As taxas de complicações tardias encontradas nos pacientes portadores de ileostomia foram maiores, se comparadas com os portadores de colostomia, principalmente com relação à dermatite periostomal. Tal diferença não foi encontrada por outros autores ${ }^{2,10}$.

\section{CONCLUSÃO}

Conclui-se que, no grupo estudado, o principal motivo da confecção de ostomia foi neoplasia de retossigmóide e canal anal. A maioria dos pacientes ostomizados é do sexo masculino, com idade média de 46.8 anos. Número elevado de complicações foi encontrado, sendo a mais comum a dermatite periestomal. Outro problema freqüente foi a má adaptação da placa de ostomia à pele.

Tabela 1 - Complicações tardias dos estomas.

\section{Tipo do estoma}

\begin{tabular}{lcrrrrr}
\cline { 2 - 6 } Complicações & \multicolumn{2}{c}{$\begin{array}{c}\text { Colostomia } \\
(\mathbf{n = 1 5 2})\end{array}$} & \multicolumn{2}{c}{$\begin{array}{c}\text { Ileostomia } \\
(\mathbf{n = 2 1})\end{array}$} & \multicolumn{2}{c}{$\begin{array}{c}\text { Urostomia } \\
(\mathbf{n = 5})\end{array}$} \\
\cline { 2 - 7 } & $\mathbf{n}$ & $\mathbf{\%}$ & $\mathbf{n}$ & $\mathbf{\%}$ & $\mathbf{n}$ & $\mathbf{\%}$ \\
\hline Dermatite & 32 & 21,1 & 13 & 61,9 & 3 & 60 \\
Estoma plano & 24 & 15,8 & 6 & 28,6 & 1 & 2 \\
Hérnia periostomal & 16 & 10,5 & 2 & 9,5 & 0 & 0 \\
Retração & 14 & 9,2 & 4 & 19,0 & 0 & 0 \\
\hline
\end{tabular}


ABSTRACT: OBJECTIVE: To elaborate the profile of the patients with permanent ostomies and the complications related to the stoma. MATERIAL AND METHODS: This is a retrospective study based on the database of the patients with permanent ostomies, registered in the 'Programa de Ostomizados do Centro de Especialidades Médicas' of the City Hall of Campo Grande, MS. RESULTS: It was evaluated 178 handbooks of these patients $(56,7 \%$ men and $43,3 \%$ women). The average age was 46,8 years for the male group and 54.6 years for the female group. It was found 152 colostomies $(85,4 \%), 21$ ileostomies $(11,8 \%)$ and 5 urostomies $(\mathbf{2 , 8 \%})$. The main indication for the stomas was the cancer $(\mathbf{4 6 , 6 \% )}$ ). The second indication was abdominal trauma $(7,3 \%)$, and third was patients with decubitus ulcer $(6,7 \%)$. Inadequate pouch placement was found in 90 patients $(50,6 \%)$. Stomal complications had been found in 103 patients $(\mathbf{5 7 , 9 \% )}$, including skin irritation $(\mathbf{2 8 , 7 \%})$, plain stoma $(\mathbf{1 8 , 6 \% )}$, peristomal hernia $(\mathbf{1 0 , 7 \%})$ and stomal retraction $(\mathbf{1 0 , 1 \% )}$. CONCLUSIONS: It was concluded that, in the group evaluated, the major indication for stomal confection was the rectosigmoid cancer and the anal cancer. The most common was the peristomal dermatitis.

Key words: ostomy, morbidity, postoperative complications, statistics \& numerical data, health profile.

\section{REFERÊNCIAS}

1. Sousa JB, Sousa ER. Complicações do fechamento de colostomia. Rev Coleg Bras Cir 1991, 12(6):240-3.

2. Duchesne JC, Wang YZ, Weintraub SL, Boyle M, Hunt JP. Stoma complications: a multivariate analysis. Am Surg 2002, 68(11):961-6.

3. Miles RM, Greene RS. Review of colostomy in a community hospital. Am Surg 1983, 48:182-6.

4. Jacob RA, Pace WG, Thomford NR. The hazard of a permanent ileostomy. Arch Surg 1969, 99:549-52.

5. Shellito PC. Complications of abdominal stoma surgery. Dis Colon Rectum 1998, 41(12):1562-72.

6. Mealy K, O'Broin E, Donohue J, Tanner A, Keane FB. Reversible colostomy - what is the outcome? Dis Colon Rectum 1996, 39:1227-31.
7. Banurra GC, Valencia CE. Cierre de ostomías: análisis comparativo de 220 casos. Rev Chil Cir 2001, 53(1):75-82.

8. Madiba TE, Mahomva O, Haffejee AA. Does type of colostomy influence outcome of colostomy closure? S Afr J Surg 1998, 36(2):57-9.

9. Ratliff CR, Scarano KA, Donovan AM. Descriptive study of periostomal complications. J WONC 2005, 32(1):33-7.

10. Mäkelä JT, Turku PH, Laitinen ST. Analysis of late stomal complications following ostomy surgery. Ann Chir Gynaecol 1997, 86(4):305-10.

Endereço para correspondência: CARLOS HENRIQUE MARQUES DOS SANTOS

Rua Aluízio de Azevedo, 606 - São Bento

79004050 - Campo Grande - MS

Fax: 33259528

e-mail: chmarques@terra.com.br 